\title{
Effects of Incomplete Information on Traffic Guidance in Intelligent Transportation Systems
}

\author{
Yufeng Chen ${ }^{\mathrm{a}}$ and Zhengtao Xiang $^{\mathrm{b}}{ }^{*}$ \\ School of Electrical and Information Engineering, Hubei University of Automotive Technology, \\ Shiyan, Hubei 442002, PR China \\ axztcyf2002@163.com, bsyxiang2000@163.com
}

\begin{abstract}
Keywords: Information feedback strategy; Traffic flow; Cellular automaton model
Abstract. The information feedback strategies for traffic guidance are important to improve the traffic capacity in Intelligent Transportation System. It assumed that all vehicles on the road are floating vehicles in the current information feedback strategies research. In reality, not all vehicles can be equipped with vehicular terminals, which are non-floating vehicles. We proposed a new strategy, Estimated Weighted Mean Velocity Feedback Strategy (EWMVFS), which can estimate the road status in the scenario with the coexistence of floating and non-floating vehicles. Simulation results show that EWMVFS can decrease the negative influence of the non-floating vehicles, which means better stability, guidance performance and practicability.
\end{abstract}

\section{Introduction}

With the interaction of roads, vehicles, drivers and environment, the traffic flow shows complex characteristics. Many traffic flow models, which are the foundation to improve the performance of Intelligent Transportation System (ITS), are proposed to describe the forming mechanism of the complex characteristics in traffic flow system. For example, the Cellular Automation (CA) models are used to describe the dynamics of traffic flow from the aspect of micro level [1-3]. In addition, traffic control is also important to improve the performance of ITS. As a typical issue in traffic control, traffic guidance in two-route scenario can dynamically allocate the traffic flows and increase the traffic capacity in ITS. Many information feedback strategies for traffic guidance were proposed in two-route scenario [4-8]. However, all of the strategies assume that all vehicles on the road are floating vehicles, which are equipped with vehicular terminals and can upload their own traffic information to the traffic control center in real time. In such idealistic complete information scenario, all traffic information of the road can be obtained. However, in reality, due to the cost and technology, not all vehicles can or will be equipped with terminals in the short term. Thus, in a very long period of time, there are two classes of vehicles on the road, floating vehicles and non-floating vehicles. Therefore, we proposed a new strategy, Estimated Weighted Mean Velocity Feedback Strategy (EWMVFS), which can estimate the road status according to the information of floating vehicles.

The rest of the paper is organized as follows. In Section 2, the NS model and the information feedback strategy are introduced. The simulation results and performance analysis are shown in Section 3. Finally, our work is concluded in Section 4.

\section{The NS model and information feedback strategy}

The NS model is a typical CA model proposed by Nagel and Schreckenberg [1]. In NS model, the road is subdivided into cells. Each cell can be occupied by one vehicle or empty. The position and the velocity of vehicle $n$ in time step $t$ is denoted by $x_{n}(t)$ and $v_{n}(t)$, respectively. $v_{\max }$ is the maximum velocity of the vehicle. The space gap between vehicle $n$ and vehicle $n+1$ is $d_{n}(t)=x_{n+l}(t)-x_{n}(t)-1$. The parallel update rules consist of four steps. (1) Acceleration: $v_{n}(t+1)=\min \left(v_{n}(t)+1, v_{\max }\right)$. (2) Deceleration: $v_{n}(t+1)=\min \left(d_{n}(t), v_{n}(t+1)\right)$. (3) Randomization with probability $p$ :
if $(\operatorname{rand}()<p)$ then $v_{n}(t+1)=\max \left(v_{n}(t+1)-1,0\right)$. (4) Vehicle motion: $x_{n}(t+1)=x_{n}(t)+v_{n}(t+1)$. 
Two-route scenario is proposed by Wahle et al. [4]. The two routes are same in the scenario. There is an information board at the entrance, which can display the traffic guide information according to the information feedback strategy. When a vehicle arrives at the entrance at each time step, it will select one route. Vehicles can be put into two types: dynamic vehicles and static vehicles. Dynamic vehicles follow the guidance on the information board, while static vehicles select one route randomly. The density of dynamic vehicles is termed as $S_{\mathrm{dyn}}$. The density of floating vehicles is termed as $S_{\mathrm{flt}}$.

In simulation, the two-route scenario with one entrance and one exit is used [8]. If two vehicles want to go out at one time step, they have to compete for the priority for going out. And the loser will move to the last cell of the route. Meanwhile, based on the realistic dataset, Laval and Leclercq [9] found that drivers can behave aggressive or timid and the same driver may behave different at different time. $p_{\text {agg }}$ is the aggressive probability of drivers at the exit.

With the above strategies, an assumption is that all vehicles on the road are floating vehicles, which means the traffic control center can obtain all traffic information of the road. However, in reality, there are two classes of vehicles on the road, floating vehicles and non-floating vehicles. In such incomplete information scenario, the cells on the road can be classified into three categories: a cell occupied by a floating vehicle, a cell occupied by a non-floating vehicle and an empty cell. The control center cannot distinguish a cell occupied by a non-floating vehicle from an empty cell, which means that the center will treat the cell occupied by a non-floating vehicle as an empty cell. If we used the traditional feedback strategies, the guidance information cannot reflect the real status of roads. To reduce the negative influence, we proposed a new information feedback strategy, Estimated Weighted Mean Velocity Feedback Strategy (EWMVFS), which is based on our previous strategy, WMVFS [8]. With EWMVFS, the floating vehicles upload the velocity and position information to the control center, and the center will compute the estimated weighted mean velocity of the road.

$$
\tilde{V}_{w}=\frac{1}{L} \sum_{i=1}^{L}\left(k \times \frac{L-x_{i}}{L}+b\right) \times \tilde{v}_{i},
$$

where $\tilde{v}_{i}$ is the velocity of one cell. In EWMVFS, to reduce the error induced by non-floating vehicles, no matter the cell is empty or is occupied by a non-floating vehicle, the current velocity of the cell is estimated by the velocity of the floating vehicle passing by the cell recently. At time step $t$, the computation of $\tilde{v}_{i}$ of the position $x_{i}$ will obey the following rules. (1) If there is one floating vehicle $n$ at position $x_{i}$, the value of $\tilde{v}_{i}$ is the velocity of the vehicle $n$, i.e., $\tilde{v}_{i}=v_{n}(t)$. (2) Otherwise, the value of $\tilde{v}_{i}$ is the velocity of the floating vehicle, which is the last vehicle to pass by the cell $x_{i}$. Suppose the floating vehicle passes by the cell $x_{i}$ at time step $t-a$, thus we can get $\tilde{v}_{i}=v_{n}(t-a)$.

\section{Simulation result and discussion}

In our simulation, the two-route scenario with one entrance and one exit is used. The length of one cell is $7.5 \mathrm{~m}$ and $L=2000$ cells. Thus we get the length of the each route is $15 \mathrm{~km}$. Other configurations are: $v_{\max }=3, p=0.25, p_{\text {agg }}=0.6, S_{\mathrm{dyn}}=0.5, S_{\mathrm{flt}}=\{0.9,0.5\}$.

Static vehicles can be divided into static floating vehicles and static non-floating vehicles. Static non-floating vehicles will select routes randomly. However, for static floating vehicles, buses can be regarded as a typical class because buses are often equipped with vehicular terminals and will follow fixed public transportation routes. Suppose most of the public transportation routes pass through route A, thus buses prefer to route A. Other static floating vehicles, such as some private vehicles with vehicular terminals and ignoring guide on the information board, will select routes randomly.

For static floating vehicles, the probability to select route $\mathrm{A}$ is termed as $S_{\mathrm{d} \_\mathrm{a}}$. However, for different $S_{\mathrm{flt}}, S_{\mathrm{d} \_ \text {a }}$ will be assigned to different values. For example, in the first stage of the development of floating vehicles, the value of $S_{\text {flt }}$ is small because private vehicles are rarely equipped with terminals and most of static floating vehicles are buses. In such period, $S_{\mathrm{d} \_ \text {a }}$ will be larger because we suppose that buses prefer to select route A. With the increase of floating vehicles, the value of $S_{\mathrm{flt}}$ becomes larger and larger, more and more private vehicles become floating vehicles, the percentage of private 
vehicles in static floating vehicles becomes larger and larger, and thus the value of $S_{\mathrm{d} \_a}$ becomes smaller and smaller. In this paper, according to the value of $S_{\mathrm{flt}}, S_{\mathrm{d} \_\mathrm{a}}$ is assigned to different values, as $S_{\mathrm{d}_{\mathrm{a}} \mathrm{a}}=$ $\{0.6,0.8\}$ corresponding to $S_{\mathrm{flt}}=\{0.9,0.5\}$.

In simulation, both routes are empty at the beginning. Vehicles at the entrance select one route randomly before the 100th time step. From the 101st time step, dynamic vehicles select one route according to the information feedback strategy. The data are recorded from the 40000th to 50000th time step. The performance metrics are vehicle number $N$, mean velocity $V_{\text {mean }}$ and travel time $T$.

When $S_{\mathrm{flt}} \neq 1$, i.e., an incomplete information scenario, the performances of TTFS, MVFS, CCFS are much worse than that of WMVFS and EWMVFS. Thus we will compare the performance between WMVFS and EWMVFS in the incomplete information scenario. The simulation results of vehicle number $N$, mean velocity $V_{\text {mean }}$ and travel time $T$ of WMVFS and EWMVFS with $S_{\text {flt }}=\{0.9,0.5\}$ are shown in Fig. 1 to Fig. 3.
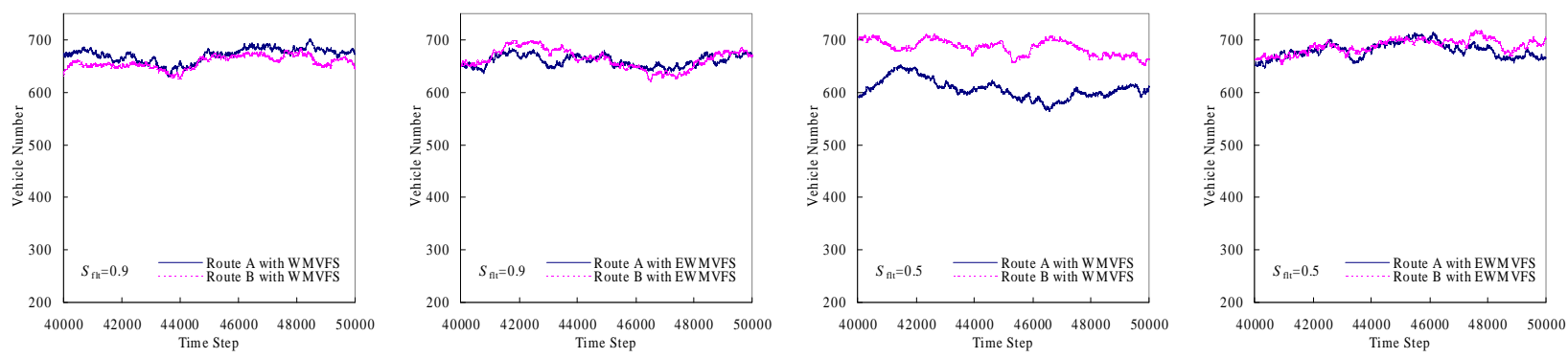

Fig. 1. Vehicle number $N$ of WMVFS and EWMVFS with different $S_{\mathrm{flt}}$.
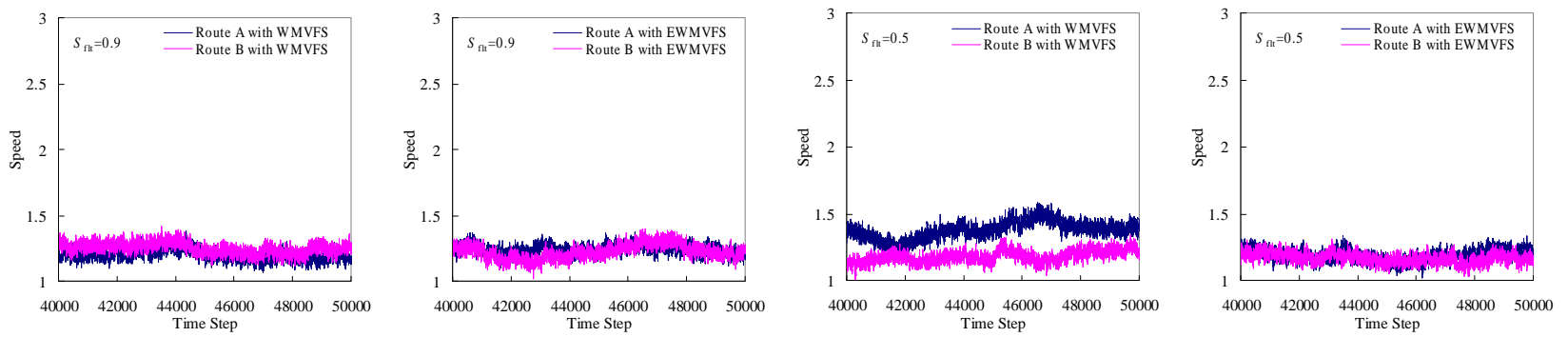

Fig. 2. Mean velocity $V_{\text {mean }}$ of WMVFS and EWMVFS with different $S_{\text {flt }}$.
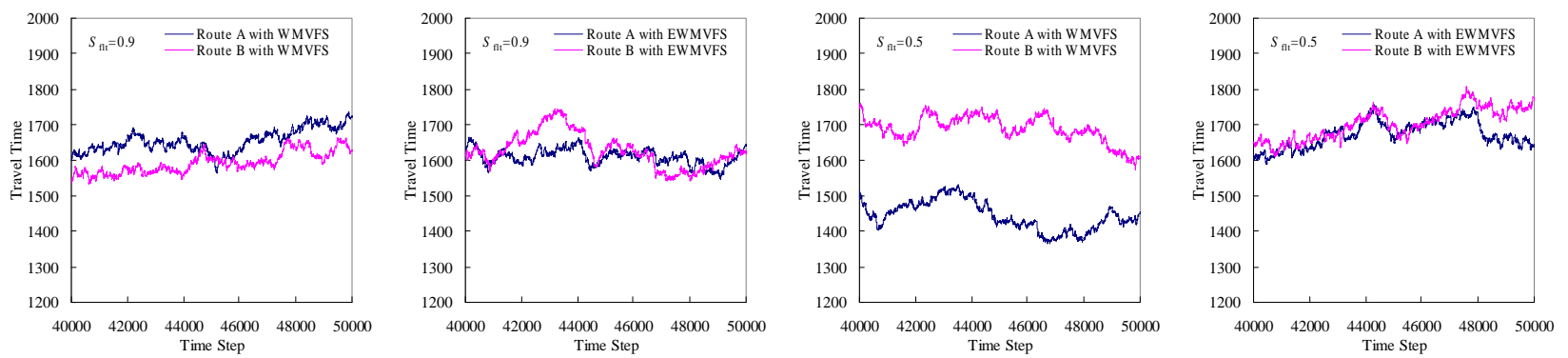

Fig. 3. Travel time $T$ of WMVFS and EWMVFS with different $S_{\mathrm{flt}}$.

In the fig. 1 to fig. 3 , when the percentage of floating vehicles is high, for example, $S_{\mathrm{flt}}=0.9$, the performances of EWMVFS and WMVFS are similar. The vehicle number $N$, mean velocity $V_{\text {mean }}$ and travel time $T$ of WMVFS and EWMVFS with $S_{\text {flt }}=0.9$ have not much differences. In addition, all the metrics of the two strategies show good stability.

However, with the decrease of the percentage of floating vehicles in Fig. 1 to Fig. 3, i.e., $S_{\mathrm{flt}}=0.5$, the performance of WMVFS decreases, while EWMVFS gives stable performance, as shown below.

(1) When considering vehicle number $N$ in Fig. 1, with the decrease of the percentage of floating vehicles, the difference between the vehicle numbers of the two routes increases for WMVFS. The vehicle number of route $A$ is less than that of route $B$ for WMVFS. However, there is not much difference between the vehicle numbers of route A and route B for EWMVFS.

(2) For mean velocity $V_{\text {mean }}$ and travel time $T$, the performance comparison of WMVFS and EWMVFS shows same results as that with vehicle number. As the decrease of the percentage of floating vehicles, for WMVFS, the differences of mean velocity $V_{\text {mean }}$ and travel time $T$ between two 
routes become larger. In Fig. 2, the mean velocity $V_{\text {mean }}$ of route $\mathrm{A}$ are higher than that of route $\mathrm{B}$, and in Fig. 3, the travel time $T$ of route A are less than that of route B. However, for EWMVFS, the mean velocity $V_{\text {mean }}$ and travel time $T$ of the two routes are similar.

In general, when considering the metrics of vehicle number, mean velocity and travel time, the performance difference between the two strategies is much. For WMVFS, it takes more time for vehicles to pass through route $B$ than route $A$. And more vehicles select route $B$, which means more vehicles select wrong route. For EWMVFS, the time costs of route A and route B are similar, and the numbers of vehicles selecting route $\mathrm{A}$ and route $\mathrm{B}$ are similar, too. From the angle of drivers, they prefer to select the less congested road with less travel time based on the feedback information. Thus, when considering the requirements of drivers, EWMVFS performs better than WMVFS.

\section{Conclusions}

Current research of information feedback strategies for traffic guidance focuses on the floating vehicles. In such scenario, the control center can obtain the complete information on roads. However, limited by the development of the Internet of vehicles, floating vehicles and non-floating vehicles will coexist. In this paper, the influence of non-floating vehicles on the typical information feedback strategies is analyzed. And the EWMVFS is proposed based on the WMVFS, can estimate the road status according to the information of floating vehicles and is applicable to the scenario of incomplete traffic information. Simulation results show that EWMVFS can decrease the negative influence of the non-floating vehicles, which means better stability, guidance performance and practicability.

\section{Acknowledgements}

This work is financially supported by National Sci-Tech Support Plan of the Ministry of Science and Technology of China (No. 2014BAG01B03, 2014BAG01B02), Key Program of the Natural Science Foundation of Hubei Province of China (No. 2013CFA054), General Program of the Natural Science Foundation of Hubei Province of China (No. 2014CFB287), Key Program of Science and Technology of Hubei Provincial Department of Education (No. D20141804), Intelligent Driving Control Key Laboratory of Hubei Province (Hubei University of Automotive Technology, preparing) (No. ZDK2201403).

\section{References}

[1] K. Nagel, M. Schreckenberg, A cellular automaton model for freeway traffic, J. Phys. I, 2 (1992) 2211-2229.

[2] B.S. Kerner, S.L. Klenov, D.E. Wolf, Cellular automata approach to three-phase traffic theory, J. Phys. A: Math. Gen., 35 (2002) 9971-10013.

[3] Z.T. Xiang, Y.J. Li, Y.F. Chen, L. Xiong, Simulating synchronized traffic flow and wide moving jam based on the brake light rule, Physica A, 392 (2013), 5399-5413.

[4] J. Wahle, A.L.C. Bazzan, F. Klugl, M. Schreckenberg, Decision dynamics in a traffic scenario, Physica A, 287 (2000) 669-681.

[5] K. Lee, P.M. Hui, B.H. Wang, N.F. Johnson, Effects of announcing global information in a two-route traffic flow model, J. Phys. Soc. Jpn., 70 (2001) 3507-3510.

[6] W.X. Wang, B.H. Wang, W.C. Zheng, C.Y. Yin, T. Zhou, Advanced information feedback in intelligent traffic systems, Phys. Rev. E, 72 (2005) 066702.

[7] C.F. Dong, X. Ma, B.H. Wang, Weighted congestion coefficient feedback in intelligent transportation systems, Phys. Lett. A, 374 (2010) 1326-1331.

[8] Z.T. Xiang, L. Xiong, A weighted mean velocity feedback strategy in intelligent two-route traffic systems, Chin. Phys. B, 22 (2013) 028901.

[9] J.A. Laval, L. Leclercq, A mechanism to describe the formation and propagation of stop-and-go waves in congested freeway traffic, Phil. Trans. R. Soc. A, 368 (2010) 4519-4541. 